\title{
A cognitive characterization of dyscalculia in Turner syndrome
}

\author{
Marie Bruandet $^{\mathrm{a}, \mathrm{b}}$, Nicolas Molko ${ }^{\mathrm{a}}$, Laurent Cohen ${ }^{\mathrm{a}, \mathrm{c}}$, Stanislas Dehaene $^{\mathrm{a}, *}$ \\ a INSERM Unit 562 “Cognitive Neuroimaging”, Service Hospitalier Frédéric Joliot, \\ CEA/DRM/DSV, 4 Place du général Leclerc, 91401 Orsay Cedex, France \\ b Service des Urgences Cérébro-Vasculaires, Hôpital de la Salpêtrière, AP-HP, Paris, France \\ ${ }^{\mathrm{c}}$ Institut de Neurologie, Hôpital de la Salpêtrière, AP-HP, Paris, France
}

Received 5 December 2002; received in revised form 11 August 2003; accepted 29 August 2003

\begin{abstract}
Current theories of number processing postulate that the human abilities for arithmetic are based on cerebral circuits that are partially laid down under genetic control and later modified by schooling and education. This view predicts the existence of genetic diseases that interfere specifically with components of the number system. Here, we investigate whether Turner syndrome (TS) corresponds to this definition. TS is a genetic disorder which affects one woman in 2500 and is characterized by partial or complete absence of one X chromosome. In addition to well-characterized physical and hormonal dysfunction, TS patients exhibit cognitive deficits including dyscalculia. We tested 12 women with Turner syndrome and 13 control subjects on a cognitive battery including arithmetical tests (addition, subtraction, multiplication, division) as well as tests of the understanding of numerosity and quantity (cognitive estimation, estimation, comparison, bisection, subitizing/counting). Impairments were observed in cognitive estimation, subitizing, and calculation. We examine whether these deficits can be attributed to a single source, and discuss the possible implications of hormonal and genetic factors in the neuropsychological profile of TS patients.
\end{abstract}

(C) 2003 Elsevier Ltd. All rights reserved.

Keywords: Developmental dyscalculia; Turner Ulrich syndrome; Estrogen; X chromosome; Parietal

\section{Introduction}

What are the origins of human arithmetical abilities? Although arithmetic is, to a large extent, a cultural construction acquired at school, an elementary sense of numbers has been proposed to be part of the genetic endowment of our species (Dehaene, 1997). Several arguments support this hypothesis. First, the ability to recognize the approximate numerosity of a collection and to incorporate it into elementary calculations is present in all cultures, emerges in the first year of life, and has precursors in various animal species (Dehaene, Dehaene-Lambertz, \& Cohen, 1998). Furthermore, brain imaging methods have revealed that calculation relies on a reproducible cerebral network. In particular, a small area of the left and right intraparietal sulci is systematically activated whenever numerical quantities are manipulated (Dehaene, Piazza, Pinel, \& Cohen, 2003). Recently, a possible homolog of this area has been identified in the intraparietal sulcus of macaque monkeys, further strengthen-

\footnotetext{
* Corresponding author.

E-mail address: dehaene@shfj.cea.fr (S. Dehaene).
}

ing the hypothesis that number sense in humans has a long evolutionary history (Sawamura, Shima, \& Tanji, 2002).

Our working hypothesis, therefore, is that the basic layout of neural circuitry in the parietal lobe is under genetic control and provides the human infant with the ability to attend to the numerosity parameter of sets in their environment. Later epigenetic enhancements of this representation and its connection to other circuits for language or the visual recognition of Arabic symbols would provide a basis for children's acquisition of the cultural tools of arithmetic.

If this model is correct, then it should be possible to identify genetic defects or mutations that perturb the laying down of parietal lobe circuits and interfere with the normal development of arithmetic, resulting in developmental dyscalculia. Developmental dyscalculia, defined as a disproportionate deficit in calculation and arithmetic, is a relatively frequent deficit (3-6\% of children) which can be observed either in conjunction with reading and/or attention disorders, or in isolation (Kosc, 1974; Sokol, Macaruso, \& Gollan, 1994; Spellacy \& Peter, 1978). Two recent papers have observed clear impairments of the left parietal cortex in children with developmental dyscalculia associated with prematurity (Isaacs, Edmonds, Lucas, \& Gadian, 2001) or 
of unknown origin (Levy, Reis, \& Grafman, 1999). In those cases, no link to a genetic defect could be made. However, the hypothesis that at least some of the dyscalculias are of genetic origin is strengthened by the finding that dyscalculia is often found in several members of the same family (Shalev et al., 2001).

In the present paper, we investigate the possibility that children with Turner syndrome (TS) suffer from a genetically determined impairment of cerebral circuits involved in the cognitive manipulation of numbers. TS is a genetic disease that affects one in 2500 girls and is due to the partial or total deletion of one $\mathrm{X}$ chromosome (long arm isochromosome, circular chromosome, partial deletion). Although the phenotype of TS may vary with the genotype and the mosaicity, it is mainly characterized by well-documented physical features (short stature, webbing of the neck, abnormal carrying angle of the elbow), and abnormal estrogen production and pubertal development. The treatment of TS includes growth factor supplementation in childhood and, after the normal age of puberty, estrogen and progesterone supplementation. Patients with Turner syndrome also have moderate cognitive impairments, which include deficits in visual memory, visual-spatial and attentional tasks, and social relations, in the context of a normal verbal IQ (Rovet, 1993) and normal or even superior language and reading skills (Temple \& Carney, 1996). Most interestingly in the present context is the documentation of a mild to severe deficit in mathematics, particularly clear in arithmetic (Mazzocco, 1998; Temple \& Marriott, 1998). Brain imaging studies suggest that this may be related to a bilateral parieto-occipital dysfunction. A positron emission tomography study of five adult women demonstrated a glucose hypometabolism in bilateral parietal and occipital regions (Clark, Klonoff, \& Hadyen, 1990). Two anatomical MR studies also demonstrated bilateral reductions in parieto-occipital brain volume, together with several other cortical and subcortical regions (Murphy et al., 1993; Reiss et al., 1993; Reiss, Mazzocco, Greenlaw, Freund, \& Ross, 1995).

The goal of the present paper is to better characterize the dyscalculia in TS girls. Arithmetic is a complex ability which reflects the coordination of multiple cerebral networks that vary with task demands (Dehaene \& Cohen, 1995). Current cognitive theories distinguish at least two subcomponents in the mental manipulation of number (Dehaene, Spelke, Stanescu, Pinel, \& Tsivkin, 1999). Some operations, such as multiplication and exact addition rely on arithmetic tables stored in rote verbal memory and engage left-hemispheric language circuits in the angular gyrus and inferior prefrontal cortices. Other operations, such as comparison, subtraction and approximate addition, are not learned by rote and are solved by quantity manipulations that rely on the bilateral intraparietal sulci (Chochon, Cohen, van de Moortele, \& Dehaene, 1999; Lee, 2000). The quantity and verbal systems are anatomically contiguous in the left parietal lobe and interact closely during any calculation (Stanescu-Cosson et al., 2000). Thus, it is important to clarify which of those sys- tems, if any, is responsible for the arithmetic impairments exhibited by TS patients.

Many studies of arithmetic impairments in TS have used standardized batteries or overall arithmetic scores, but a few have provided more specific information relevant to the present work. In the Mazzocco et al. study (Mazzocco, 1998), a broad population of 5-16-year-old children was tested with the Woodcock-Johnson-R battery. The results indicated poorer calculation scores and a greater prevalence of calculation errors in Turner syndrome relative to either fragile X patients or normal controls. Rovet et al. (Rovet, Szekely, \& Hockenberry, 1994), using the WAIS-R and the WRAT-R in 7 to 16-year-old children, observed a reduced performance in the digit span and arithmetic subtests in TS patients. Subanalyses showed impairments mainly in multidigit addition and in multiplication fact retrieval. Two more recent studies (Temple \& Marriott, 1998; Temple \& Sherwood, 2002) measured the patients' calculation time with a stopwatch during the performance of simple number processing and arithmetic problems. The results indicated that basic number processing (reading, writing, copying, and comparing numbers) was unimpaired, but that calculation (single-digit addition, single-digit multiplication, and multidigit calculation procedures) was slowed, more prone to errors, or both.

In the present study, we aimed at providing finer-grained measures of number processing and calculation in TS. Our study was designed to improve on earlier work in several respects. First, we measured the performance of TS patients using computerized tests, which permitted the measurement of response times with millisecond accuracy. Second, we studied a group of young adults, thus reducing to some extent the impact of the patient's educational difficulties. Third, as regards calculation and number comparison, we did not simply study the patients' overall response time and error rate, but also computed derivative measures that have been used in cognitive psychology to characterize normal subjects' performance. For instance, we examined separately the response to tie and non-tie problems, we measured the impact of problem size on the patients's response times, and we also measured the size of the distance effect in number comparison. Fourth, we included four new tests that were not studied in previous work. Two of them, numerical bisection and cognitive estimation, are tests of the understanding of numerical quantities that are known to be highly sensitive to both adult and developmental dyscalculia (Dehaene \& Cohen, 1997; Kopera-Frye, Dehaene, \& Streissguth, 1996). The other two new tests, subitizing and estimation, measured the ability of TS patients to evaluate numerical quantities presented in a non-symbolic format, as a set of dots. All previous studies of TS have used tests of calculation with numerical symbols, such as digits or number words. However, the extraction of numerosity from a set of dots figures amongst the most basic numerical abilities which are present in animals and in human infants, (Dehaene, 1997) and may thus be laid down early on in development, possibly under 
genetic control. It would therefore be particularly interesting if those basic functions were found impaired in a genetic disease, such as Turner syndrome.

\section{Method}

\subsection{Participants}

12 TS patients participated in the study after giving informed consent. The patients were recruited from a hospital in Paris and from contacts with the French Turner Syndrome association (AGAT). Age ranged from 18 to 40 (mean 24). All patients had a 45X karyotype (complete deletion, no mosaicity) and were treated with oestrogen since the normal age of puberty; three had received growth hormone during their youth. Note that the patients were recruited on a volunteering basis, and thus we cannot ascertain that our population is a representative sample. TS patients were matched to a control group of 13 normal subjects based on age (range 20-40, mean 26) and education. Participants were tested with four tests from the WAIS-R including two verbal tests (vocabulary and similarities tests), and two performance tests (design-picture completion and cubes tests). $T$-tests revealed that TS did not differ than controls subjects except on the vocabulary test in which controls subjects were significantly better (see Table 1). Because inclusion was on a voluntary basis, some bias in recruitment cannot be excluded. This, together with the exclusion of calculation tests from our performance measures, may explain that our TS patients score better on tests of performance IQ that is usually reported in the literature.

\subsection{Cognitive battery}

Participants were tested for $2 \mathrm{~h}$. They were seated in front of a computer at a distance of $40-50 \mathrm{~cm}$. Response times were recorded with millisecond accuracy via a voice key or two large response buttons connected to the computer, which was running the EXPE software (Pallier, Dupoux, \& Jeannin, 1997). The problems stayed on the computer's screen until the subjects answered. There were 12 tests divided into three parts: simple input-output tests (verbal counting, reading and writing digits); tests evaluating the sense of numerosity and quantity (cognitive estimation, digit comparison, bisec-

Table 1

Background performance measures for the patient (TS) and control groups

\begin{tabular}{lrllll}
\hline & TS & S.D. & Controls & S.D. & $P$ \\
\hline Age & 24 & 7 & 26 & 7 & 0.45 \\
Vocabulary test & 12 & 2 & 14 & 2 & 0.04 \\
Similarities test & 11 & 3 & 13 & 2 & 0.14 \\
Design-picture completion & 12 & 2 & 11 & 1 & 0.45 \\
Cube test & 8 & 2 & 10 & 3 & 0.13 \\
\hline
\end{tabular}

tion, estimation and subitizing); and arithmetic tests (addition, subtraction, multiplication, and division).

For the first three simple input-output tests, response times were recorded globally for the entire block of trials. The timer was manually controlled, and subjects were asked to answer as quickly as possible. For the forward and backward counting blocks, subjects had to recite the numbers from 1 up to 20 , then from 20 to 1 . For the reading block, subjects read 20 Arabic numerals 1-4 digits long $(0,3,4,6$, 9, 18, 11, 30, 74, 93, 169, 207, 472, 800, 353, 1047, 2708, $7400,4414,3293)$. For the writing block, they wrote down 20 similar Arabic numerals that were dictated by the experimenter $(1,2,5,7,8,19,12,50,76,95,138,409,673,700$, 929, 1032, 6801, 9100, 8717, 2798).

A second part examined various quantity evaluation tests.

\subsubsection{Cognitive estimation}

This test was a French translation and modification of a task previously used to demonstrate number estimation deficits in foetal alcohol syndrome (Kopera-Frye et al., 1996). Subjects were asked 30 questions whose answer is generally not known but can be approximately evaluated. (e.g. What is the length of a bus? How much does a typical chair weight?) The main variable of interest is the proportion of strange responses that betray an incorrect adequation of numerical quantity to the context. No strong speed pressure was imposed, and responses times were recorded globally for documentation purposes.

\subsubsection{Digit comparison}

Two Arabic digits were presented on the computer screen, left and right of a central fixation point. Subjects had to press a button with their left hand or their right hand to indicate the side of the larger digit as quickly as possible. There were 72 trials corresponding to all pairings of the numbers 1-9. This test reflects access to an internal quantity representation (Moyer \& Landauer, 1967).

\subsubsection{Bisection}

Two Arabic digits were presented side by side on the computer screen, with the smaller digit to the left of the larger digit. Subjects had to find as quickly as possible the digit located in the middle of the interval (e.g. when the interval is 2 and 4 , the expected response is 3 ). There were 32 trials, corresponding to two presentations of each of the pairs of numbers $1-9$ with a distance of $2,4,6$ or 8 . This test has been found to be severely impaired in a patient with acalculia and Gerstmann's syndrome following parietal lesion (Dehaene \& Cohen, 1997).

\subsubsection{Subitizing}

Subjects were asked to say aloud, as precisely as possible, the number of dots in a randomly organized square display that remained on screen until the response. They were told that the numbers ranged from 1 to 8 . This test allows the evaluation of the speed and accuracy of 'subitizing' (fast 
quantification of small numbers of dots, generally 1-3), the size of the subitizing range, and the speed and accuracy of counting beyond that range (Mandler \& Shebo, 1982). All numbers 1-8 were presented 10 times each, for a total of 80 trials. On each trial, a new random pattern of white rectangular dots was generated, with the constraints that the dots resided within a framed $200 \times 200$ voxels regions on the screen (approximately $8^{\circ}$ of visual angle), and that there was a minimal distance of eight voxels between any two dots. Dot size was fixed for a given display, but varied randomly from trial to trial between 6 and 12 voxels. Response times were recorded via a voice key, and the vocal responses were later scored for accuracy.

\subsubsection{Estimation}

A random sequence of green squares was quickly flashed on the computer screen. Subjects were asked to estimate, without attempting to count, how many squares had been presented. They were not told anything about the range of numbers tested. This test was introduced by Whalen et al. (Whalen, Gallistel, \& Gelman, 1999) to estimate the variability of non-verbal numerosity estimation in humans using a procedure similar to the one used in animals. No speed pressure was imposed, as the dependent measure was accuracy rather than response time. Individual squares were presented for $29 \mathrm{~ms}$, separated by random blank periods whose duration was a $71 \mathrm{~ms}$ plus a Poisson-distributed variable with a mean of $100 \mathrm{~ms}$. All the odd numbers between 7 and 21 were presented five times each, for a total of 40 trials.

In the third part, the four arithmetical operations were tested. Response times were recorded via the voice key and error rates were computed for each problem. All problems stayed on the computer screen until the subject answered.

\subsubsection{Addition}

All 54 additions of two digits from 0 to 9 , including ties but excluding $0+0$, were presented once. The larger digit was always presented to the left of the smaller digit (e.g. $9+6)$.

\subsubsection{Subtraction}

All 54 subtractions of the same digit pairs as in addition were presented once (e.g. $9-6$ ).

\subsubsection{Multiplication}

The same digit pairs, but this time with the larger number on the right, were presented for multiplication $($ e.g. $6 \times 9$ ).

\subsubsection{Division}

Only very simple division problems were used. The problem set comprised 24 integer divisions problems with the dividend ranging from digits from 4 to 20 , the divisor ranging from 2 to 9 , and the result ranging from 2 to 9 (e.g. $4 / 2$, 20/4)

\section{Results}

\subsection{Mean response times and error rates in each task}

Table 2 presents the mean response times and error rates in each task for the groups. Statistical comparisons indicated that the patients were always slower than controls, and that this reached significance only in reading, writing, cognitive estimation, subitizing, addition, subtraction and division. Nevertheless, the patients' performance was relatively well preserved, as indicated by low error rates $(<18 \%)$ which were significantly higher than those of the controls only in the cognitive estimation task. We now present a detailed analysis of the different tasks.

\subsection{Number processing and counting}

Neither the patients nor the controls made any errors in forward or backward counting, oral reading of Arabic numerals, and writing Arabic numerals to dictation (Table 2). This is important since it implies that our subsequent tests of calculation were not affected by basic problems in number comprehension and production. On response times, $t$-tests

Table 2

Mean response times and error rates for the experimental tasks

\begin{tabular}{|c|c|c|c|c|c|c|c|c|c|c|}
\hline & \multicolumn{5}{|c|}{ Reaction time (ms) } & \multicolumn{5}{|c|}{ Error rate $(\%)$} \\
\hline & Turners & S.D. & Controls & S.D. & $P$-value & Turners & S.D. & Control & S.D. & $P$-value \\
\hline Forward counting & 264 & 38 & 239 & 43 & NS & 0 & 0 & 0 & 0 & NS \\
\hline Backward counting & 354 & 69 & 323 & 57 & NS & 0 & 0 & 0 & 0 & NS \\
\hline Reading & 844 & 136 & 709 & 104 & 0.02 & 0 & 0 & 0 & 0 & NS \\
\hline Writing & 2258 & 329 & 1811 & 480 & 0.02 & 0 & 0 & 0 & 0 & NS \\
\hline Cognitive estimation & 12915 & 4732 & 10464 & 2617 & 0.05 & 6.4 & 0.36 & 2.6 & 0.26 & 0.05 \\
\hline Digit comparison & 521 & 108 & 493 & 53 & NS & 2.2 & 2.8 & 1.2 & 2.3 & NS \\
\hline Subitizing/counting & 1417 & 354 & 1129 & 228 & 0.01 & 2 & 3 & 1.7 & 3.3 & NS \\
\hline Bisection & 2192 & 974 & 1768 & 827 & NS & 17.9 & 20.3 & 17.8 & 18.8 & NS \\
\hline Addition & 1151 & 286 & 964 & 174 & 0.03 & 1.21 & 2 & 0.6 & 1.3 & NS \\
\hline Multiplication & 2145 & 1275 & 1554 & 615 & NS & 10 & 10 & 6 & 6.2 & NS \\
\hline Subtraction & 1336 & 339 & 1028 & 210 & 0.004 & 3 & 8.7 & 1.5 & 3.7 & NS \\
\hline Division & 1744 & 539 & 1308 & 389 & 0.02 & 2.8 & 5.5 & 1.2 & 3.2 & NS \\
\hline
\end{tabular}


indicated that TS patients were no slower than the controls in counting forward or backward $(P>0.1)$, but were moderately slower in reading numbers $(844$ versus $709 \mathrm{~ms}$ per item, $P=0.02)$ and in writing numbers (2.25 versus $1.81 \mathrm{~s}$ per item, $P=0.02$ ). Those results should be taken with caution, however, because they were derived from a manual measurement of an entire block of trials.

\subsection{Cognitive estimation}

For each numerical question, a range of approximately reasonable answers was defined a priori based on our previous work (Kopera-Frye et al., 1996). A $t$-test showed that TS patients made a significantly greater proportion of unreasonable answers than the controls (6.4 versus $2.6 \% ; P=$ $0.05)$. This was true even though the patients tended to respond more slowly than the controls (12.9 versus 10.4 s per problem, $P=0.056)$. The type of errors tended to differ between patients and controls. In the patients group, errors were frequently made on questions about length, and always in the direction of underestimation. For instance, six patients gave an answer below $4 \mathrm{~m}$ for the length of a bus. Another four patients gave an answer below $6 \mathrm{~m}$ for the size of the highest tree in the world. Control subjects erred more often on questions of general culture (e.g. the duration of Christopher Columbus' transatlantic trip).

\subsection{Number comparison}

Mean response times and error rates were calculated for each level of distance between the two numbers to be compared (Fig. 1). Because there were fewer measures for larger distances, all distances larger than or equal to 7 were grouped together. The response times were submitted to an ANOVA with group (patients or controls) and distance (7 levels) as factors. There was a main effect of distance, as both patients and controls were increasingly faster as the distance between

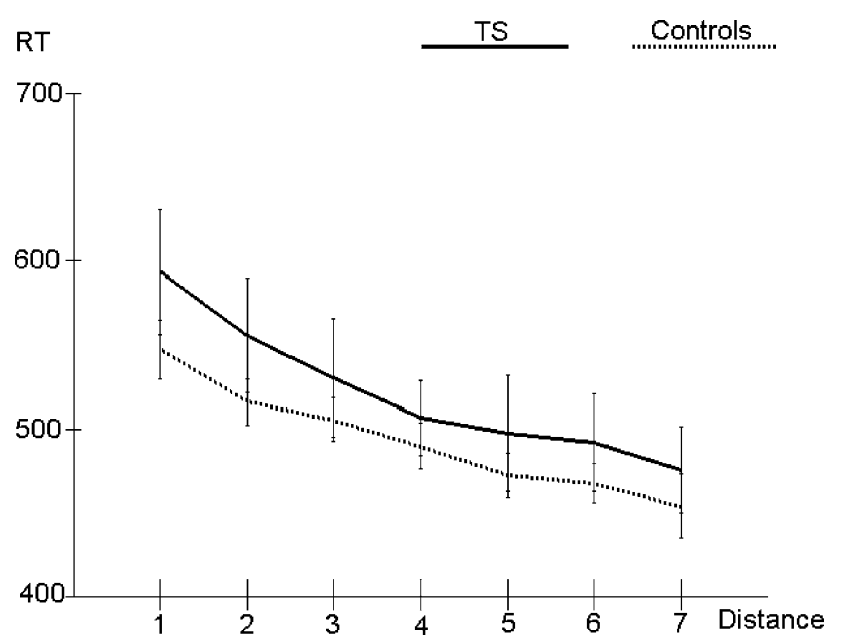

Fig. 1. Response times in number comparison as a function of the numerical distance between the compared numbers. the numbers increased $(F(6,138)=35.38, P<0.0001)$. The main effect of group was not significant $(F(1,23)=$ $0.78, P=0.38$ ), patients being only $28 \mathrm{~ms}$ slower than controls. Nor was there an interaction between group and distance $(F(6,138)=0.74, P=0.62)$. Similar results were obtained with error rates. There were more errors for close than for far digits, thus revealing a significant distance effect $(F(6,138)=12.4, P<0.001)$, but no main effect of group $(F(1,23)=2.41, P=0.13)$, nor a group by distance interaction $(F(6,138)=0.72, P=0.63)$.

\subsection{Bisection}

In the bisection test, one control subject was excluded from the analysis due to microphone failure. Mean response times from the remaining subjects were submitted to ANOVA with factors of group and the numerical interval between the bisected numbers $(2,4,6$ or 8$)$. There was no difference between patients and controls $(F(1,22)=1.75$, $P=0.19)$, and no group by interval interaction $(F(3,66)=$ 1.77, $P=0.16)$. The response times of all subjects varied similarly with distance $(F(3,66)=11.8, P<0.0001)$, first increasing from 2 to 6 , then decreasing for the single trial with a distance of 8 (interval 1-9). Note that the patients were, on average, slower than the controls (e.g. $922 \mathrm{~ms}$ slower for bisecting pairs with an interval of 6), but this did not reach significance due to a high variance in the control group. A similar ANOVA on error rates revealed a significant effect of interval $(F(3,66)=15.9, P<0.0001)$, with a profile similar to the response times curve, but again with no significant group effect or any interaction (both $F s<1$ ).

\subsection{Subitizing/counting}

Response times in the subitizing/counting task are presented in Fig. 2. Mean response times were submitted to an ANOVA with factors of group and the number of dots in

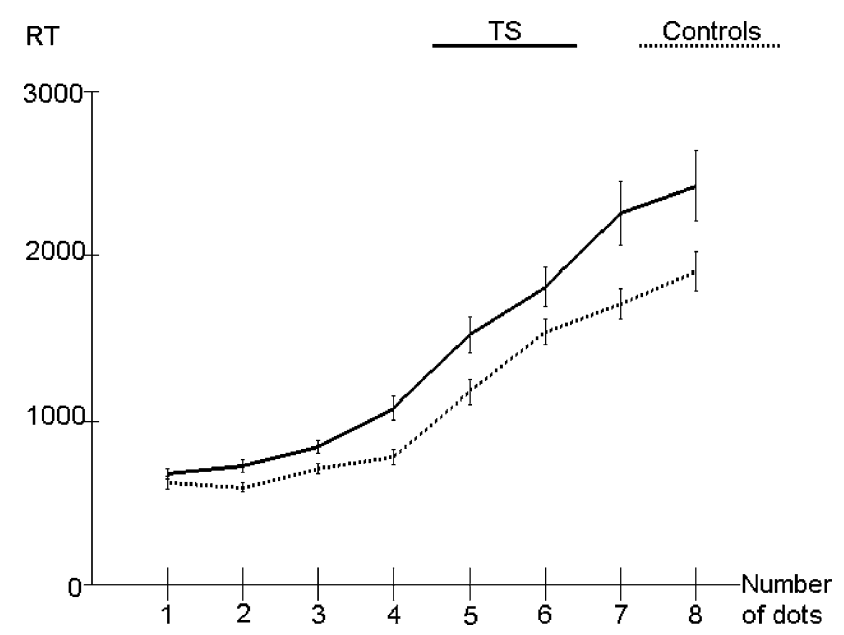

Fig. 2. Response times in the subitizing/counting task as a function of the number of dots. 
the stimulus (from 1 to 8 ). Response times increased significantly with the number of dots $(F(7,161)=166.97, P<$ 0.0001). As reported in the literature, (Mandler \& Shebo, 1982) this increase was non-linear. A significant quadratic trend $(F(1,23)=47.31, P<0.0001)$ indicated that responses times increased only moderately in the range $1-4$, and started to increase much more sharply by about $300 \mathrm{~ms}$ per number above that range (see Fig. 2). This reflects the classical distinction between subitizing, the fast apprehension of numerosities below 4 , and counting, the serial verbal process used to enumerate more than four items.

Examination of group differences revealed that subitizing, but not counting, differed between the patients and the controls. There was a main difference between the response times of patients and controls $(F(1,23)=7.42$, $P=0.012)$ and an interaction between group and numerosity $(F(7,161)=3.70, P=0.001)$. Patients and controls did not differ on numerosity $1(F(1,23)=1.31, P=0.26)$, but started to diverge significantly starting at numerosity 2 , where patients were already $133 \mathrm{~ms}$ slower than controls $(F(1,23)=7.37, P=0.012)$. In the subitizing range, the patients showed an abnormally elevated increase in response times, as assessed by the interaction of group with the linear contrast from 1 to $4(F(1,23)=9.62, P<0.005)$. This suggested that many of the patients were counting within the range in which controls normally subitize. Indeed, the increase in response time from 3 to 4 dots was $237 \mathrm{~ms}$ in the patients, close to the value observed in the counting range, where it was only $72 \mathrm{~ms}$ in the controls, a significance difference $(F(1,23)=9.42, P=0.005)$. For numbers above 4 , in the counting range, response times increased linearly with numerosity and there was no difference in the slope of this increase between patients and controls $(F(1,23)=$ $1.95, P=0.17)$.

A similar ANOVA on error rates showed that the two populations did not differ, neither on overall error rate $(F(1,23)<1)$ nor on the group by numerosity interaction $(F(7,161)=1.77 P=0.09)$. All subjects made more errors when the numerosity increased $(F(7,161)=5.61$, $P<0.0001)$.

\subsection{Estimation}

Several measures were derived from subjects' estimates of the numerosity of a random series of flashes. First, we examined the subjects' mean numerical response to each of the presented numerosities, from 7 to 21 flashes (see Fig. 3). An ANOVA revealed that although responses increased with numerosity overall $(F(1,154)=364.28$, $P<0.0001)$, the patients did not differ from controls subjects overall $(F(1,23)=2.73, P=0.11)$ and there was no group by numerosity interaction $(F(7,154)=1.54$, $P=0.15)$. Second, examination of the mean correlation coefficient between subjects' mean responses and input numerosity revealed no difference between groups (mean correlation coefficient $=0.99$ in both groups). Third, we

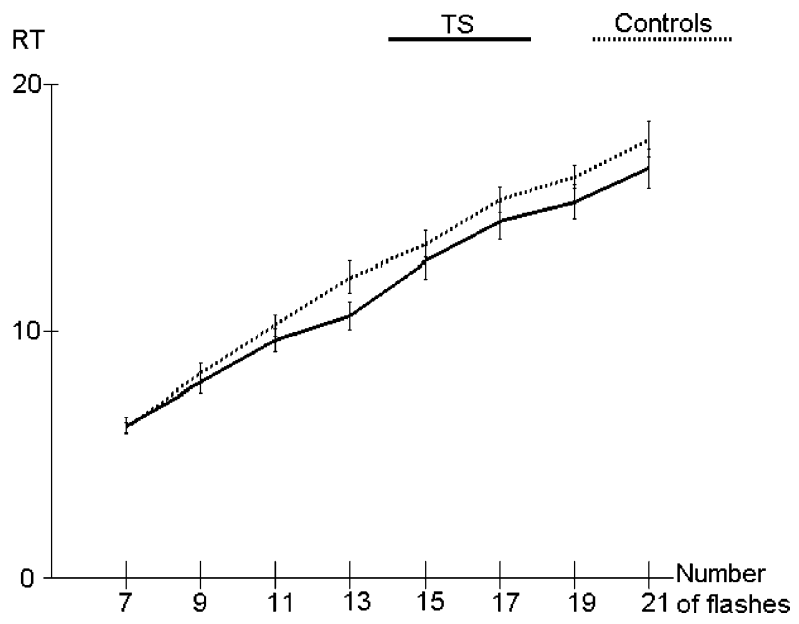

Fig. 3. Mean numerical response of the patients and the controls when asked to estimate the numerosity of a fast sequence of flashes.

measured the standard deviation (S.D.) of response to each numerosity. As reported by others, (Whalen et al., 1999) S.D. increased with numerosity $(F(7,161)=3.86, P<$ 0.0001 , but there was again no overall difference between groups $(F(7,23)=0.83, P=0.37)$, nor any interaction $(F(7,161)=0.52, P=0.82)$. Fourth, we computed the coefficient of variation, which is the standard deviation divided by the mean response to each numerosity. The coefficient of variation was essentially constant for all numerosities (mean $=0.13$ ), though there was a marginal decrease with numerosity $(F(7,161)=2.01, P=0.056)$. Again, there was no difference between groups $F(1,23)=0, P=0.96$ ) nor any interaction $(F(7,161)=0.49, P=0.84)$. Finally, as expected from those results, the Weber fraction, which is the average coefficient of variation did not differ in the two groups (0.13 for both).

\subsection{Addition}

Response times for addition and the other three arithmetic operations are presented in Fig. 4. For the purpose of statistical analysis, addition problems were divided into four categories: problems with identical digits (ties), problems that can be solved by a rule $(n+0=n)$, small problems (with a sum smaller than 10), and large problems (with a sum of 10 or more). An ANOVA on mean response times (RT) between the four categories and the two groups was computed. It revealed a significant group effect. TS patients were slower than the control group $(F(1,23)=4.800, P=0.03)$. RTs from the two groups were significantly different between the four categories $(F(3,69)=76.52, P<0.0001)$. Finally, there was a significant interaction between category and group $(F(3,69)=3.55, P=0.0188)$. Comparisons within each category indicated that the patients did not differ from the controls on ties problems $(F(1,23)=0.33$, $P=0.57)$, but were slower than controls on rules-based problems $(F(1,23)=4.50, P=0.044)$, small problems 

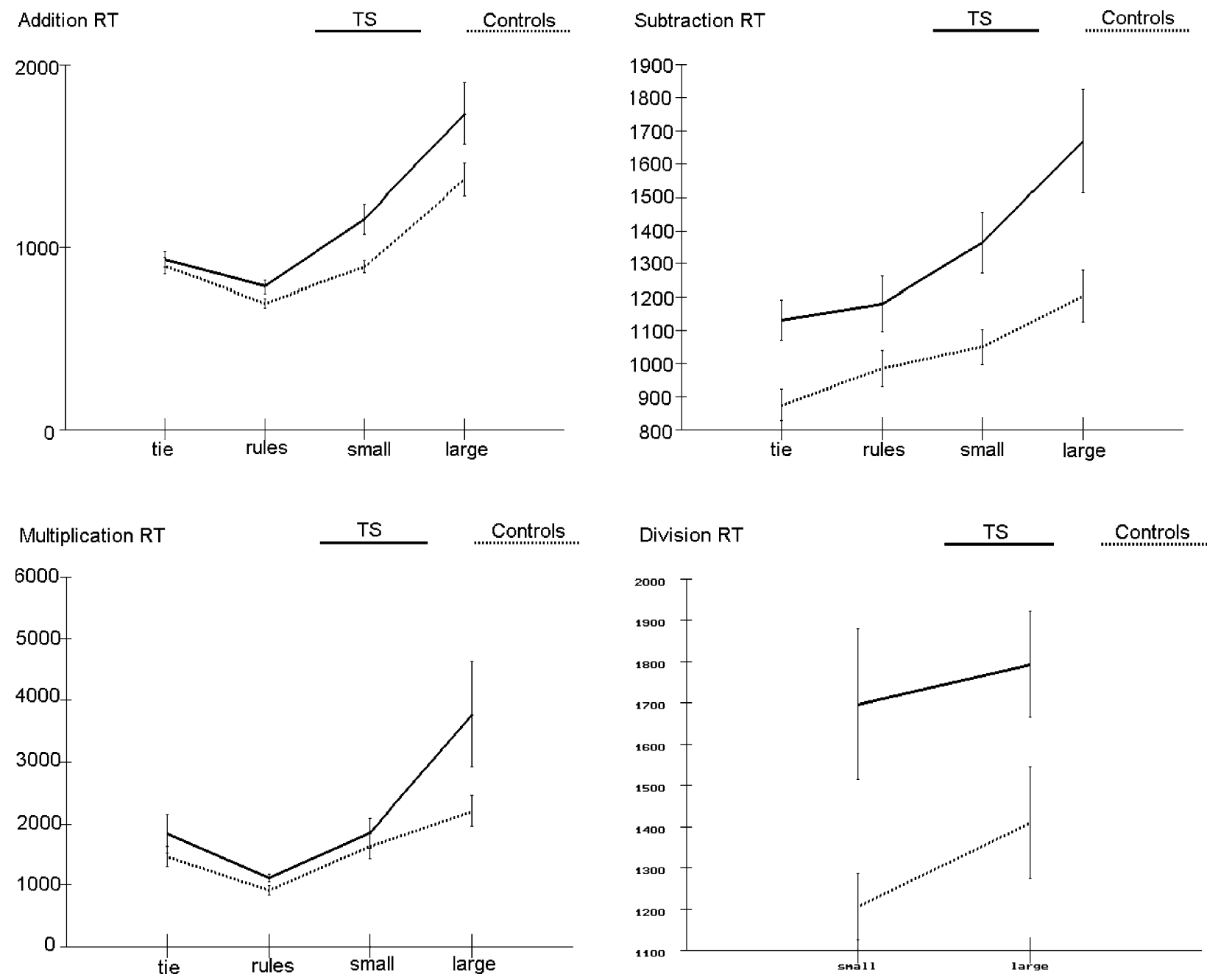

Fig. 4. Response times in the four calculation tasks as a function of problem type.

$(F(1,23)=9.36, P=0.006)$, and marginally on larger problems $(F(1,23)=3.69, P=0.067)$.

The statistical analysis of error rates revealed an overall difference between the four types of problems $(F(3,69)=$ $6.67, P=0.0005$ ), indicating more frequent errors for small and large categories than for tie or rule-based problems. However, neither the main effect of group, nor the interaction between group and category were significant (respectively $F(1,23)=1.22, P=0.28$, and $F<1)$.

\subsection{Subtraction}

Four categories of subtraction problems were compared: ties $(n-n)$, problems that can be solved by a rule $(n-0=$ $n$ ), small problems (in which the digit to be subtracted was 6 or less) and large problems (in which the digit to be subtracted was 7, 8 or 9). An ANOVA on mean response times found that patients were $307 \mathrm{~ms}$ slower than the controls $(F(1,23)=9.83, P=0.004)$. Response times varied with the category $(F(3,69)=25.5, P=0.001)$, and were especially slower when the digit to be subtracted was larger than six. This effect tended to be more prominent in the TS patients, but the interaction between the group and category factors failed to reach significance $(F(3,69)=2.50$, $P=0.066$ ). A similar ANOVA on mean error rate yielded no significant effects.

\subsection{Multiplication}

Four categories of multiplication problems were compared: ties $(n \times n)$, problems that could be solved by a rule $(n \times 0=0, n \times 1=n)$, small problems (in which the first digit was 4 or less), and large problems (in which the first digit was larger than 4). An ANOVA on mean response times showed that the patients were globally $591 \mathrm{~ms}$ slower than controls, but this effect failed to reach conventional levels of significance $(F(1,23)=3.11, P=0.091)$. The group by category interaction also fell short of significance $(F(3,69)=2.53, P=0.064)$. Examination of the response time curves (Fig. 4) showed that the patients were considerably slower that the patients by $1569 \mathrm{~ms}$ on large multiplication problems only, but even this difference did 
not reach significance due to a large variance in the patient group $(F(1,23)=3.35, P=0.080)$.

A similar ANOVA on error rates revealed a main effect of category, indicating that errors were nearly exclusively concentrated in the large category $(F(3,69)=17.44$ $P<0.0001)$. However, there was no main effect of group $(F(1,23)=2.18 P=0.15)$, nor any interaction between group and category $(F(3,69)=1.36, P=0.26)$.

\subsection{Division}

Because of the small number of division trials, only two categories were defined for the statistical analysis: divisions by 2 and division by a number larger than 2 . An ANOVA revealed that patients were significantly slower than controls by $437 \mathrm{~ms}(F(1,23)=6.01, P=0.02)$. There was a main effect of problem category, as both patients and controls were slower with the divisions by a number larger than $2(F(1,23)=4.98, P=0.035)$. However, no group by category interaction was found $(F(1,23)=0.61, P=0.44)$. On error rates, none of the effects reached significance $(P>$ $0.2)$.

\section{Discussion}

Our goal was to examine the performance of TS patients in simple tests designed to study the elementary cognitive mechanism of number in normal subjects. The results revealed the following cognitive profile of dyscalculia in TS. First, no problems were found in simple comprehension and production of numbers. Oral counting, reading and writing were normal. Second, no significant impairment was detected in three of five tests of quantity processing: number comparison, numerosity estimation, and bisection. Only subitizing and cognitive estimation were impaired. Subitizing in patients was restricted to smaller numerosities than in controls, resulting in an earlier onset of counting. The cognitive estimation task resulted in a greater number of anomalous responses in TS than in controls. Third, TS patients were slower in all arithmetic tasks except multiplication, and showed a greater impairment as the numbers increased. It was a general characteristic of our results that the impairments manifested themselves mostly as increased response times rather than elevated error rates.

Our results fit well with previous studies of arithmetic in TS. Preservation of basic number processing (reading and writing numbers), relative to impaired arithmetic, was reported previously by several groups (Mazzocco, 1998; Temple \& Marriott, 1998; Temple \& Sherwood, 2002). Temple and Sherwood (Temple \& Sherwood, 2002), although studying a small sample of six TS children and measuring RTs with a stopwatch, found that, as in our young adult group, performance was slower in addition and subtraction, but not in multiplication (though their patients did make more errors than controls in multiplication). Furthermore, in a simple addition task, Temple and Marriott (Temple \& Marriott, 1998) report that TS patients were increasingly slower as the numbers increased, resulting in a elevated numerical size effect compared to controls, similar to what we found. It is likely that, as the numbers increase, the subjects have to rely increasingly on multidigit calculation strategies, which have been shown to be reduced in TS patients (Mazzocco, 1998; Rovet et al., 1994; Temple \& Marriott, 1998). Indeed, our results indicate that TS patients behave essentially normally on simple arithmetic problems, that are thought to be solved by rote verbal memory or by elementary rules. Addition ties (e.g. $2+2$ ), as well as multiplication ties $(3 \times 3)$, rule-based (e.g. $n \times 0)$, and small multiplication problems (e.g. $2 \times 3$ ), were solved with a normal speed and error rate. This suggests that the simple verbal storage of arithmetic facts is not impaired in TS, a hypothesis that would fit with their preserved comprehension and production of numbers, their intact verbal counting, and their generally normal verbal IQ.

The impairment observed in arithmetic respected the gradient generally attributed to impaired quantity processing (Dehaene \& Cohen, 1997; Dehaene et al., 2003), with subtraction being globally impaired, addition being impaired for small and large problems (but not rule-based problems), and multiplication showing no significant impairment. Research with normal subjects indicates that large addition are generally not solved by direct memory retrieval, but rather by the application of various strategies, often involving semantic mental manipulations of numbers (e.g. solving 15-6 as (15-5)-1 by noticing that 6 is close to 5) (Dehaene \& Cohen, 1997; Dehaene et al., 2003; LeFevre, Sadesky, \& Bisanz, 1996; LeFevre et al., 1996). In fMRI, this increased demand on "semantic elaboration strategies" (Dehaene \& Cohen, 1995) is reflected in an increased activation in parietal, frontal, and cingulate areas as the size of the operands of an addition problem increases (Stanescu-Cosson et al., 2000). It seems that the design and/or the application of such strategies was particularly slow and difficult for TS patients. Little is known about the resolution of simple division problems, but the global slowing down of TS patients with this operation, even with problems that were matched to the simple multiplication facts that they could solve (e.g. 4:2), suggests that a similar component of semantic strategical processing may be involved and may be impaired in TS.

The patients' reduced ability to resolve non-rote arithmetic problems could potentially arise from either a reduced capacity for semantic understanding of numbers, or from a more general inability to execute any complex strategy. As noted above, TS patients are known to exhibit executive and working memory impairments (Ross, Roeltgen, Feuillan, Kushner, \& Cutler, 1998, 2000; Ross et al., 2002; Temple \& Marriott, 1998). Although those functions were not tested in our patients, they might presumably account for their calculation deficits. However, we also found basic deficits in number processing that do not seem so easily reducible to an executive or working memory dysfunction. 
Most notably, subitizing was subtly impaired: TS patients resorted to counting earlier than the controls, suggesting that they had a reduced ability to immediately identify a small numerosity. It is notable that counting itself, as assessed by the amount of increase of response time with numerosity in the counting range of 4-8 dots, was normal. This suggests that there might be an impairment in quantity processing, over and above any strategical processing impairment. The errors in cognitive estimation, occasionally resulting in grossly incorrect responses, such as $3 \mathrm{~m}$ for the length of a bus, also testify to a basic problem in relating number symbols to quantities in the external world.

A difficulty for this interpretation, however, is that three other tests thought to tap quantity processing were not significantly impaired. TS patients could decide which of two digits is the largest, with a normal response time (confirming an earlier report (Temple \& Sherwood, 2002)) and a normal distance effect. They could bisect a numerical interval with the same accuracy as controls. Finally they could estimate the numerosity of a sequence of visual flashes, with an accuracy comparable to normal subjects. In explaining those results, at least three non-exclusive possibilities must be considered.

First, some of our tests might be insensitive. This is a likely possibility for the bisection and estimation tests. In bisection, TS patients did not perform well, and were on average slower than the controls. However, no significance differences were observed because several normal controls also found this test quite difficult, especially for an interval of 4 or 6 (e.g. bisect the interval 2-8), and because performance was thus extremely variable in both controls and patients. Similarly, in estimation, we only collected five numerical estimates for each target numerosity, which may have made it difficult to detect a subtle impairment. A trend towards underestimation was present in the patients, but did not reach significance. Furthermore, response times, which were the only sensitive measure in most calculation subtests, were not collected during numerosity estimation.

Second, patients were tested in adulthood, at a point when considerable developmental compensation could have taken place. We consider this a likely possibility for the good performance of the number comparison task. Even in adults with acquired lesions, this test rarely shows important deficits, and they are often quickly compensated (Dehaene \& Cohen, 1997; Delazer \& Butterworth, 1997). It seems likely that, should some of our subjects have suffered from an initial difficulty with a capacity as basic as number comparison, it would have been the target of much training and rehabilitation during their years of schooling, so that no deficit would be visible in adulthood. This seems possible because overall the numerical deficits in TS are rather mild and concern response time more than error rate, so that they are presumably accessible to drilling.

A third possibility, finally, is that the preserved tests point to a partially preserved quantity system. It is conceivable that subitizing, estimation, and comparison tests rely on par- tially dissociable subsystems of quantity knowledge, and in particular that subitizing relies on a small-number system specifically concerned with the numbers 1,2 and 3 . This possibility requires further investigation.

Our investigation of TS patients was motivated by the hypothesis that this genetic disease could affect some of the genes responsible for an early biasing of the parietal lobe for quantity processing. While this hypothesis remains tenable, we would like to conclude by stressing the complex intrication of genetic and hormonal mechanisms in Turner's syndrome. The complete deletion of one $\mathrm{X}$ chromosome results in an absence of estrogens throughout infancy and childhood, before the patients are treated with estrogen at the normal time of puberty. The hormonal deficit itself could be a critical factor in cognitive development. Estrogens are known to enhance working memory performance in menopausal women (Haberecht et al., 2001; Keenan, Ezzat, Ginsburg, \& Moore, 2001; Sherwin, 1997, 1998). Shaywitz et al (Shaywitz et al., 1999) have shown with 46 post-menopausal women that estrogen intake modulates the activation of the inferior parietal lobule, increasing it during storage of verbal material and decreasing it during storage of non-verbal material. Furthermore, estrogens improve visuo-spatial memory in ovariectomized rhesus monkeys (Lacreuse, Wilson, \& Herndon, 2002) and in rats (Lund et al., 2001). Conversely, knockout mice for the estrogen beta receptor fail to learn spatial tasks (Rissman, Heck, Leonard, Shupnik, \& Gustafsson, 2002). In Turner's syndrome, Ross et al. (Ross et al., 1998, 2000) have studied the cognitive effects of estrogen therapy started early on in childhood (7-9 years old). They found benefits in verbal and non-verbal memory. In particular, backward digit span and recall of the Rey figure were better in TS patients treated with estrogens than in untreated patients, although performance did not reach the level attained by normal female controls. Both tasks are known to involve the parietal lobes. Thus, the results suggest that parietal impairment may be partially, but not totally compensated by estrogen replacement therapy, perhaps pointing to an early effect of estrogen on parietal lobe organization. Various neurobiological mechanisms support this possibility. The presence of estrogen receptors in the cortex suggests a local action of this hormone (Shughrue \& Merchenthaler, 2000). In ovariectomized rats, the formation of new excitatory synapses is induced by estradiol and involves the participation of a neurotransmitter, glutamic acid, acting through NMDA receptors (Woolley \& McEwen, 1994). Estrogens can also modify attentional and memory processes indirectly through their known influence on cholinergic, noradrenergic, and dopaminergic systems (McEwen, 1999).

There are at least two other biological factors that further complicate the interpretation of TS impairments. First, women suffering from TS are often treated with growth hormone, which could perhaps influence cognitive functions. The current suggestion is that growth hormone replacement in TS does not improve the neuropsychological profile, even when this therapy is started early in childhood (Ross, 
Feuillan, Kushner, Roeltgen, \& Cutler, 1997). In our study, comparisons of treated and untreated patients also did not reveal any significant differences, although the groups were too small to draw any strong conclusion. Second, TS patients often have variable karyotypes. Though we tested only monosomic $45, \mathrm{X}$ patients, many TS patients have a "mosaic" karyotype, with only a variable percentage of cells showing the partial or complete deletion of an X chromosome. Most studies suggest that such mosaic subtypes are generally less impaired than monosomic $45, \mathrm{X}$ patients (Temple \& Carney, 1995). However, this is challenged by a recent study in which cognitive impairments were not influenced by the mosaic characteristics (Ross et al., 2002). Another open issue concerns the influence of genetic imprinting on the cognitive phenotype in TS. Skuse et al. (Skuse et al., 1997) suggest that TS patients whose remaining X chromosome originates from the father show milder executive dysfunctions than those who inherited their remaining $\mathrm{X}$ chromosome from the mother. Unfortunately, such parental information was not available for our patients.

\section{Conclusion}

Our study confirms that women suffering from Turner syndrome exhibit impairments in calculation and number processing, possibly pointing to a left parietal dysfunction. However, the impairments are variable and relatively mild when tested in adulthood. Furthermore, the genetic mechanisms of the disease are clouded by inter-individual variability and by the effects of hormone replacement therapy. Further insight into the genetic predisposition of the human species for number processing may come from detailed neuroimaging studies of TS patients, possibly with partial rather than complete $\mathrm{X}$ chromosome deletions, as well as of other genetic diseases, such as fragile X (Kwon et al., 2001; Mazzocco, 1998). Naturally, the discovery of a family with a monogenic impairment in numerical abilities, comparable to the recently described KE family with inherited speech and language disorders (Lai, Fisher, Hurst, Vargha-Khadem, \& Monaco, 2001; Watkins et al., 2002), would permit much faster progress.

\section{References}

Chochon, F., Cohen, L., van de Moortele, P. F., \& Dehaene, S. (1999). Differential contributions of the left and right inferior parietal lobules to number processing. Journal of Cognitive Neuroscience, 11, 617-630.

Clark, C., Klonoff, H., \& Hadyen, M. (1990). Regional cerebral glucose metabolism in Turner syndrome. Canadian Journal of Neurological Sciences, 17, 140-144.

Dehaene, S. (1997). The number sense. New York: Oxford University Press.

Dehaene, S., \& Cohen, L. (1995). Towards an anatomical and functional model of number processing. Mathematical Cognition, 1, 83-120.

Dehaene, S., \& Cohen, L. (1997). Cerebral pathways for calculation: Double dissociation between rote verbal and quantitative knowledge of arithmetic. Cortex, 33, 219-250.
Dehaene, S., Dehaene-Lambertz, G., \& Cohen, L. (1998). Abstract representations of numbers in the animal and human brain. Trends in Neuroscience, 21, 355-361.

Dehaene, S., Piazza, M., Pinel, P., \& Cohen, L. (2003). Three parietal circuits for number processing. Cognitive Neuropsychology, 20, 487506.

Dehaene, S., Spelke, E., Stanescu, R., Pinel, P., \& Tsivkin, S. (1999). Sources of mathematical thinking: Behavioral and brain-imaging evidence. Science, 284, 970-974.

Delazer, M., \& Butterworth, B. (1997). A dissociation of number meanings. Cognitive Neuropsychology, 14, 613-636.

Haberecht, M. F., Menon, V., Warsofsky, I. S., White, C. D., Dyer-Friedman, J., \& Glover, G. H. (2001). Functional neuroanatomy of visuo-spatial working memory in Turner syndrome. Human Brain Mapping, 14(2), 96-107.

Isaacs, E. B., Edmonds, C. J., Lucas, A., \& Gadian, D. G. (2001). Calculation difficulties in children of very low birthweight: A neural correlate. Brain, 124(Pt 9), 1701-1707.

Keenan, P. A., Ezzat, W. H., Ginsburg, K., \& Moore, G. J. (2001). Prefrontal cortex as the site of estrogen's effect on cognition. Psychoneuroendocrinology, 26(6), 577-590.

Kopera-Frye, K., Dehaene, S., \& Streissguth, A. P. (1996). Impairments of number processing induced by prenatal alcohol exposure. Neuropsychologia, 34, 1187-1196.

Kosc, L. (1974). Developmental dyscalculia. Journal of Learning Disabilities, 7, 165-177.

Kwon, H., Menon, V., Eliez, S., Warsofsky, I. S., White, C. D., \& Dyer-Friedman, J. (2001). Functional neuroanatomy of visuospatial working memory in fragile $\times$ syndrome: Relation to behavioral and molecular measures. American Journal of Psychiatry, 158(7), 10401051.

Lacreuse, A., Wilson, M. E., \& Herndon, J. G. (2002). Estradiol, but not raloxifene, improves aspects of spatial working memory in aged ovariectomized rhesus monkeys. Neurobiology Aging, 23(4), 589600.

Lai, C. S., Fisher, S. E., Hurst, J. A., Vargha-Khadem, F., \& Monaco, A. P. (2001). A forkhead-domain gene is mutated in a severe speech and language disorder. Nature, 413(6855), 519-523.

Lee, K. M. (2000). Cortical areas differentially involved in multiplication and subtraction: A functional magnetic resonance imaging study and correlation with a case of selective acalculia. Annals of Neurology, 48 , $657-661$.

LeFevre, J., Sadesky, G. S., \& Bisanz, J. (1996). Selection of procedures in mental addition: Reassessing the problem size effect in adults: Learning, Memory, and Cognition. Journal of Experimental Psychology, 22, 216-230.

LeFevre, J. A., Bisanz, J., Daley, K. E., Buffone, L., Greenbaum, S. L., \& Sadesky, G. S. (1996). Multiple routes to solution of single-digit multiplication problems. Journal of Experimental Psychology: General, $125,284-306$

Levy, L. M., Reis, I. L., \& Grafman, J. (1999). Metabolic abnormalities detected by H-MRS in dyscalculia and dysgraphia. Neurology, 53, 639-641.

Lund, T. D., West, T. W., Tian, L. Y., Bu, L. H., Simmons, D. L., \& Setchell, K. D. (2001). Visual spatial memory is enhanced in female rats (but inhibited in males) by dietary soy phytoestrogens. BMC Neuroscience, 2(1), 20.

Mandler, G., \& Shebo, B. J. (1982). Subitizing: An analysis of its component processes. Journal of Experimental Psychology: General, $111,1-21$.

Mazzocco, M. M. (1998). A process approach to describing mathematics difficulties in girls with Turner syndrome. Pediatrics, 102(2 Pt 3), 492-496.

McEwen, B. S. (1999). Clinical review 108: The molecular and neuroanatomical basis for estrogen effects in the central nervous system. Journal of Clinical Endocrinology Metabolism, 84(6), 17901797. 
Moyer, R. S., \& Landauer, T. K. (1967). Time required for judgements of numerical inequality. Nature, 215, 1519-1520.

Murphy, D. G., DeCarli, C., Daly, E., Haxby, J. V., Allen, G., \& White, B. J. (1993). X-chromosome effects on female brain: A magnetic resonance imaging study of Turner's syndrome. Lancet, 342(8881), 1197-1200 [see comments].

Pallier, C., Dupoux, E., \& Jeannin, X. (1997). Expe5: An expandable programming language for on-line psychological experiments, methods, instruments and computers. Behavior Research, 29, 322327.

Reiss, A. L., Freund, L., Plotnick, L., Baumgardner, T., Green, K., \& Sozer, A. C. (1993). The effects of X monosomy on brain development: Monozygotic twins discordant for Turner's syndrome. Annals of Neurology, 34(1), 95-107.

Reiss, A. L., Mazzocco, M. M., Greenlaw, R., Freund, L. S., \& Ross, J. L. (1995). Neurodevelopmental effects of X monosomy: A volumetric imaging study. Annals of Neurology, 38(5), 731-738.

Rissman, E. F., Heck, A. L., Leonard, J. E., Shupnik, M. A., \& Gustafsson, J. A. (2002). Disruption of estrogen receptor beta gene impairs spatial learning in female mice. Proceedings of the National Academy of Sciences of the United States of America, 99(6), 39964001.

Ross, J. L., Feuillan, P., Kushner, H., Roeltgen, D., \& Cutler Jr., G. B. (1997). Absence of growth hormone effects on cognitive function in girls with Turner syndrome. Journal of Clinical Endocrinology Metabolism, 82(6), 1814-1817.

Ross, J. L., Roeltgen, D., Feuillan, P., Kushner, H., \& Cutler Jr., G. B. (1998). Effects of estrogen on nonverbal processing speed and motor function in girls with Turner's syndrome. Journal of Clinical Endocrinology Metabolism, 83(9), 3198-3204.

Ross, J. L., Roeltgen, D., Feuillan, P., Kushner, H., \& Cutler Jr., G. B. (2000). Use of estrogen in young girls with Turner syndrome: Effects on memory. Neurology, 54(1), 164-170.

Ross, J. L., Stefanatos, G. A., Kushner, H., Zinn, A., Bondy, C., \& Roeltgen, D. (2002). Persistent cognitive deficits in adult women with Turner syndrome. Neurology, 58(2), 218-225.

Rovet, J. F. (1993). The psychoeducational characteristics of children with Turner syndrome. Journal of Learning Disabilities, 26(5), 333341.

Rovet, J. F., Szekely, C., \& Hockenberry, M. N. (1994). Specific arithmetic calculation deficits in children with Turner syndrome. Journal of Clinical Experimental Neuropsychology, 16(6), 820-839.

Sawamura, H., Shima, K., \& Tanji, J. (2002). Numerical representation for action in the parietal cortex of the monkey. Nature, 415(6874), 918-922.

Shalev, R. S., Manor, O., Kerem, B., Ayali, M., Badichi, N., \& Friedlander, Y. (2001). Developmental dyscalculia is a familial learning disability. Journal of Learning Disabilities, 34, 59-65.
Shaywitz, S. E., Shaywitz, B. A., Pugh, K. R., Fulbright, R. K., Skudlarski, P., \& Mencl, W. E. (1999). Effect of estrogen on brain activation patterns in postmenopausal women during working memory tasks. JAMA, 281(13), 1197-1202.

Sherwin, B. B. (1997). Estrogen effects on cognition in menopausal women. Neurology, 48(5 Suppl 7), S21-26.

Sherwin, B. B. (1998). Estrogen and cognitive functioning in women. Proceedings of the Society of Experimental Biological Medicine, 217(1), 17-22.

Shughrue, P. J., \& Merchenthaler, I. (2000). Estrogen is more than just a "sex hormone": Novel sites for estrogen action in the hippocampus and cerebral cortex. Front Neuroendocrinology, 21(1), 95-101.

Skuse, D. H., James, R. S., Bishop, D. V., Coppin, B., Dalton, P., \& Aamodt-Leeper, G. (1997). Evidence from Turner's syndrome of an imprinted X-linked locus affecting cognitive function. Nature, 387(6634), 705-708 [see comments].

Sokol, S. M., Macaruso, P., \& Gollan, T. H. (1994). Developmental dyscalculia and cognitive neuropsychology. Developmental Neuropsychology, 10, 413-441.

Spellacy, F., \& Peter, B. (1978). Dyscalculia and elements of the developmental Gerstmann syndrome in school children. Cortex, 14, 197-206.

Stanescu-Cosson, R., Pinel, P., van de Moortele, P.-F., Le Bihan, D., Cohen, L., \& Dehaene, S. (2000). Cerebral bases of calculation processes: Impact of number size on the cerebral circuits for exact and approximate calculation. Brain, 123, 2240-2255.

Temple, C. M., \& Carney, R. (1996). Reading skills in children with Turner's syndrome: An analysis of hyperplexia. Cortex, 32(2), 335345.

Temple, C. M., \& Carney, R. A. (1995). Patterns of spatial functioning in Turner's syndrome. Cortex, 31(1), 109-118.

Temple, C. M., \& Marriott, A. J. (1998). Arithmetic ability and disability in Turner's syndrome: A cognitive neuropsychological analysis. Developmental Neuropsychology, 14, 47-67.

Temple, C. M., \& Sherwood, S. (2002). Representation and retrieval of arithmetical facts: Developmental difficulties. The Quartely journal of experimental psychology A. Human Experimental Psychology, 55(3), 733-752.

Watkins, K. E., Vargha-Khadem, F., Ashburner, J., Passingham, R. E., Connelly, A., \& Friston, K. J. (2002). MRI analysis of an inherited speech and language disorder: Structural brain abnormalities. Brain, 125(Pt 3), 465-478.

Whalen, J., Gallistel, C. R., \& Gelman, R. (1999). Non-verbal counting in humans: The psychophysics of number representation. Psychological Science, 10, 130-137.

Woolley, C. S., \& McEwen, B. S. (1994). Estradiol regulates hippocampal dendritic spine density via an $N$-methyl-D-aspartate receptor-dependent mechanism. Journal of Neuroscience, 14(12), 7680-7687. 